Le Lait, 1987, 67 (3), 379-392

\title{
Effets respectifs du stade de lactation, du stade de gestation et du niveau de production sur la lipolyse spontanée du lait de vache
}

\author{
Marie-Paule CHAZAL*, Y. CHILLIARD ** (1) \\ * Institut Technique de l'Elevage Bovin \\ ** INRA, Laboratoire de la Lactation, Theix \\ 63122 Ceyrat, France
}

\section{Résumé}

Les teneurs en AGL immédiatement après la traite (AGL initiaux) et après $22 \mathrm{~h}$ de stockage à $4{ }^{\circ} \mathrm{C}$ (AGL22) ont été déterminées dans le lait du soir de 211 vaches de 3 troupeaux, 2 à 3 fois au cours de la lactation. L'augmentation des teneurs en AGL initiaux au cours de lactation n'est liée qu'à la diminution du niveau de production laitière. Les teneurs en AGL22 augmentent après 12 semaines et surtout après 32 semaines de lactation. La première augmentation est due au stade de lactation luimême, mais la seconde aux effets combinés du stade de gestation avancé (plus de 24 semaines) et du faible niveau de production (moins de $5 \mathrm{~kg}$ à la traite du soir). Ces résultats sont comparables à ceux d'un autre essai (CHAZAL et CHILliaRD, 1986) portant sur un seul troupeau de 57 vaches suivies plus fréquemment (14 à 20 fois) au cours de la lactation. Toutefois, dans la présente étude l'effet du niveau de production est plus important que celui du stade de gestation, alors que l'inverse était observé dans l'autre essai. Cette différence est probablement liée à la structure des populations étudiées (nombres d'animaux et de prélèvements par animal) dans chacun des deux essais.

Mots clés: Acides gras libres - Lipolyse spontanée - Acidité de la matière grasse Stade de lactation - Stade de gestation - Niveau de production - Lait de vache.

\section{Summary}

Effects of stage of lactation, stage of pregnancy and evening milk yield on spontaneous lipolysis in bovine milk

The free fatty acid (FFA) levels after milking (initial FFA) and after $22 \mathrm{~h}$ storage at $4{ }^{\circ} \mathrm{C}$ (FFA-22) were determined in the evening milk, 2-3 times during lactation. 211 cows from 3 herds were used. The milk FFA contents were measured by a miniaturized BDI method.

(1) Adresser la correspondance à Y. Chilliard. 
The initial FFA contents were higher in milk from one herd as opposed to the others. This was because of the higher somatic cell counts in the former. The FFA-22 contents were lower in milk from the herd where the interval between morning and evening milkings was longer than for the others.

The milk inital FFA and FFA-22 contents increased during lactation. The increase in milk initial FFA content was only related to the decreased milk yield. The milk FFA-22 content increased after 12 weeks, and further, after 32 weeks of lactation. The first increase was due to the stage of lactation itself. The second increase, in late lactation, was due to the combined effects of the advanced stage of pregnancy $(>24$ weeks) and the low evening milk yield $(<5 \mathrm{~kg})$. The milk FFA-22 content increased when the evening milk yield decreased, whatever the stage of pregnancy, but especially after 24 weeks.

These results are comparable to those of another trial in which milks from 57 cows in another herd were sampled more frequently (14 to 20 times) during lactation (Chazal and Chilliard, 1986). However, in the present study, the effect of milk production level was higher than that of pregnancy stage, contrary to what was observed in the preceding trial. This difference is probably linked to the nature of the samples studied in the two experiments (number of cows and number of samples per rowl.

Key words : Free fatty acids - Spontaneous lipolysis - Fat acidity - Lactation stage Pregnancy stage - Milk yield - Bovine milk.

\section{Introduction}

La lipolyse spontanée du lait peut être définie comme l'hydrolyse de la matière grasse du lait stocké au froid, en absence de toute activation mécanique. Les facteurs physiologiques qui influencent la lipolyse spontanée sont nombreux (Jellema, 1980 ; Chilliard, 1982). Parmi ceux-ci, l'effet du stade de lactation est bien connu (Freeden et al., 1951; Jensen, 1959; Ahrne et Bjorck, 1985 ; Chazal et Chilliard, 1986). Il existe toutefois de grandes variations individuelles : la lipolyse peut augmenter fortement en fin de lactation chez certains animaux, mais ne pas évoluer chez d'autres (FrEeden et al,, 1951 ; Jensen, 1959 ; Chazal et Chilliard, 1986). Or le stade de lactation est toujours mesuré en interaction avec d'autres facteurs, dont le stade de gestation et le niveau de production laitière. Dans un premier essai, sur 57 vaches Montbéliardes d'un même troupeau suivies régulièrement au cours d'une année, un effet prépondérant du stade de gestation sur la lipolyse a été mis en évidence pour la première fois, et les parts respectives du stade de lactation, du stade de gestation et du niveau de production ont été analysées (CHAZAL et Chilliard, 1986). La présente étude, portant sur un plus grand nombre de vaches de races différentes (Montbéliardes, Frisonnes et Holstein), provenant de 3 autres troupeaux, a pour objectif de confirmer, dans des conditions différentes, cette éventuelle influence du stade de gestation, et de préciser ses interactions avec les effets du stade de lactation et du niveau de production. 


\section{Matériel et méthodes}

\section{A. Animaux}

Des prélèvements de laits individuels ont été effectués dans 2 troupeaux INRA (Marcenat, Cantal ; Theix, Puy-de-Dôme) ainsi que dans le troupeau du lycée agricole de Bourg-en-Bresse (Ain). Les caractéristiques de ces troupeaux sont présentées dans le tableau 1. Les échantillons de lait ont été prélevés 2 à 3 fois pour chaque vache, dont 1 fois pendant l'hiver à l'étable (février-mars 1984 ) et 1 fois au printemps au pâturage (mai-juin 1984).

Les animaux recevaient à volonté les fourrages présentés dans le tableau 1. Du tourteau de soja-colza tanné était distribué aux vaches du troupeau de Theix en complément de l'ensilage de maïs. En hiver à l'étable, une partie des animaux du troupeau de Bourg-en-Bresse recevait du lactosérum, et l'ensemble des drêches de brasserie. Dans les 3 troupeaux, du concentré était distribué individuellement en complément de la ration de base.

\section{B. Prélèvements de lait et analyse des échantillons}

Les prélèvements ont été effectués à la traite du soir, le lait du soir étant plus sensible à la lipolyse que le lait du matin (SUhren et al., 1981). L'échantillon a été prélevé dans le pot trayeur (Marcenat, Theix durant I'hiver) ou à l'aide d'un compteur à lait, de marque Vaikato, placé juste après la griffe, lorsque la traite était faite en salle de traite (Bourg-en-Bresse, Theix au printemps et en été). Immédiatement après la traite de chaque vache, un échantillon de $18 \mathrm{ml}$ a été thermisé à $60{ }^{\circ} \mathrm{C}$ pendant 30 minutes pour détruire la lipase du lait, puis congelé à $-20^{\circ} \mathrm{C}$ jusqu'au dosage des acides gras libres (AGL initiaux). Un $2^{\mathrm{e}}$ échantillon de $18 \mathrm{ml}$ a été stocké à $4{ }^{\circ} \mathrm{C}$ pendant 22 heures, puis thermisé et congelé jusqu'au dosage des acides gras libres (acides gras libres après stockage au froid, AGL22). Les concentrations en AGL ont été mesurées par une variante miniaturisée de la méthode BDI (Bureau of Dairy Industry) (Chazal et al., 1984). Les résultats sont exprimés en milliéquivalents pour $100 \mathrm{~g}$ de matière grasse (meq/100 g MG).

Un autre échantillon de $30 \mathrm{ml}$ de lait a été conservé avec du bichromate de potassium pendant 1 à 3 jours à $4{ }^{\circ} \mathrm{C}$, jusqu'au dosage du taux butyreux par spectrophotométrie infra-rouge (Milkoscan avec filtre A à Theix et Marcenat, et filtre B à Bourg-en-Bresse, Foss Electric, Danemark), et des cellules somatiques par comptage automatique (Fossomatic, Foss Electric, Danemark).

\section{Analyses statistiques}

L'analyse statistique des résultats a été réalisée par analyse de variance et de covariance (SEEBECK, 1973), après transformation logarithmique des teneurs en AGL pour obtenir une distribution normale des données. Pour chaque analyse statistique, l'effet du facteur «troupeau " a été pris en compte, et le taux butyreux considéré comme covariable. Les données présentées dans le tableau 2 sont les moyennes des valeurs non transformées et non ajustées. 
TABleau 1

Caractéristiques des troupeaux expérimentaux et des prélèvements Characteristics of experimental herds and samples

\begin{tabular}{|c|c|c|c|}
\hline $\begin{array}{l}\text { Troupeau } \\
\text { Herd }\end{array}$ & $\begin{array}{l}\text { INRA } \\
\text { Theix }\end{array}$ & $\begin{array}{l}\text { INRA } \\
\text { Marcenat }\end{array}$ & $\begin{array}{c}\text { L.E.P.A. } \\
\text { Bourg-en-Bresse }\end{array}$ \\
\hline \multicolumn{4}{|l|}{$\begin{array}{l}\text { Animaux } \\
\text { Animals }\end{array}$} \\
\hline $\begin{array}{l}\text { Nombre } \\
\text { Number }\end{array}$ & 65 & 100 & 46 \\
\hline $\begin{array}{l}\text { Numéro de lact. } \\
\text { Lactation number }\end{array}$ & $4,0 \pm 2,3$ & $2,8 \pm 1,9$ & $2,3 \pm 1,2$ \\
\hline $\begin{array}{l}\text { Race } \\
\text { Breed }\end{array}$ & $\begin{array}{l}\text { Frisonne } \times \text { Holstein } \\
\text { Friesian } \times \text { Holstein }\end{array}$ & $\begin{array}{l}\text { Montbéliarde (58) } \\
\text { Frisonne }(42) \\
\text { Montbéliarde (56) } \\
\text { Friesian (42) }\end{array}$ & $\begin{array}{l}\text { Montbéliarde } \\
\text { Montbéliarde }\end{array}$ \\
\hline $\begin{array}{l}\text { Période de vêlage } \\
\text { Calving period }\end{array}$ & $\begin{array}{l}\text { Octobre-mars } \\
\text { October-march }\end{array}$ & $\begin{array}{l}\text { Novembre-avril } \\
\text { November-april }\end{array}$ & $\begin{array}{c}\text { Août-février } \\
\text { August-february }\end{array}$ \\
\hline $\begin{array}{l}\text { Fourrages } \\
\text { Forages }\end{array}$ & & & \\
\hline $\begin{array}{l}\text { Hiver } \\
\text { Winter }\end{array}$ & $\begin{array}{c}\text { Ensilage maïs } \\
\text { Corn silage }\end{array}$ & $\begin{array}{c}\text { Ensilage maïs } \\
+ \text { regain } \\
\text { Corn silage }+ \text { second } \\
\text { cut hay }\end{array}$ & $\begin{array}{l}\text { Ens. maïs + ens. } \\
\text { herbe } \\
\text { Corn silage + grass } \\
\text { silage }\end{array}$ \\
\hline $\begin{array}{l}\text { Eté } \\
\text { Summer }\end{array}$ & $\begin{array}{l}\text { Pâturage } \\
\text { Pasture }\end{array}$ & $\begin{array}{c}\text { Pâturage } \\
\text { Pasture }\end{array}$ & $\begin{array}{c}\text { Pâturage } \\
\text { Pasture }\end{array}$ \\
\hline \multicolumn{4}{|l|}{$\begin{array}{l}\text { Prélèvements } \\
\text { Samples }\end{array}$} \\
\hline $\begin{array}{l}\text { Méthode } \\
\text { Method }\end{array}$ & $\begin{array}{c}\text { Hiver : pot trayeur } \\
\text { Eté : compteur à lait } \\
\text { (Vaikato) } \\
\text { Winter: bucket } \\
\text { Summer: milk } \\
\text { counter }\end{array}$ & $\begin{array}{l}\text { Pot trayeur } \\
\text { Bucket }\end{array}$ & $\begin{array}{l}\text { Compteur à lait } \\
\text { (Vaïkato) } \\
\text { Milk counter }\end{array}$ \\
\hline $\begin{array}{l}\text { Heures de traite } \\
\text { Milking time }\end{array}$ & 7 h -15 h 30 & $6 \mathrm{~h} 30-16 \mathrm{~h}$ & $6 \mathrm{~h} 30-17 \mathrm{~h}$ \\
\hline $\begin{array}{l}\text { Nombre } \\
\text { Number }\end{array}$ & 138 & 210 & 108 \\
\hline $\begin{array}{l}\text { Stade (semaines) de : } \\
\text { Stage (weeks) of : } \\
\text { Lactation } \\
\text { Lactation } \\
\text { Gestation } \\
\text { Pregnancy }\end{array}$ & $\begin{array}{l}29 \pm 12 \\
11 \pm 12\end{array}$ & $\begin{array}{l}22 \pm 11 \\
9 \pm 10\end{array}$ & $\begin{array}{l}27 \pm 9 \\
15 \pm 9\end{array}$ \\
\hline $\begin{array}{l}\text { Traite du soir : } \\
\text { Evening milking: } \\
\text { Production de lait }(\mathrm{kg}) \\
\text { Milk yield }(\mathrm{kg}) \\
\text { Nombre de cellules } \\
(\times 1000) \\
\text { Somatic cell count } \\
(\times 1000) \\
\text { Taux butyreux }(\mathrm{g} / 1) \\
\text { Fat content }(\mathrm{g} / \mathrm{l})\end{array}$ & $\begin{array}{l}6,9 \pm 3,0 \\
905 \pm 407\end{array}$ & $\begin{aligned} 7,7 & \pm 2,7 \\
276 & \pm 654\end{aligned}$ & $\begin{array}{l}8,4 \pm 2,5 \\
289 \pm 609 \\
40,3 \pm 5,4\end{array}$ \\
\hline
\end{tabular}




\section{Résultats}

\section{A. Teneurs en AGL du lait selon le troupeau et le stade de lactation (analyse I. tabl. 2)}

Sur l'ensemble des prélèvements, les teneurs en AGL initiaux des laits individuels du troupeau de Theix sont supérieures $(\mathrm{P}<0,05)$ à celles observées dans les troupeaux de Marcenat et de Bourg-en-Bresse. Elles augmentent régulièrement au cours de la lactation dans les 3 troupeaux.

Les teneurs en AGL22 des laits individuels du troupeau de Bourg-enBresse sont plus faibles $(\mathrm{P}<0,05)$ que celles observées à Theix et à Marcenat. Elles augmentent dans les 3 troupeaux après 12 semaines de lactation $(\mathrm{P}<0,05)$ et surtout après 32 semaines $(\mathrm{P}<0,05)$, mais avec de grandes variations individuelles (coefficient de variation de $87 \%$ après 32 semaines contre $32 \%$ avant 12 semaines).

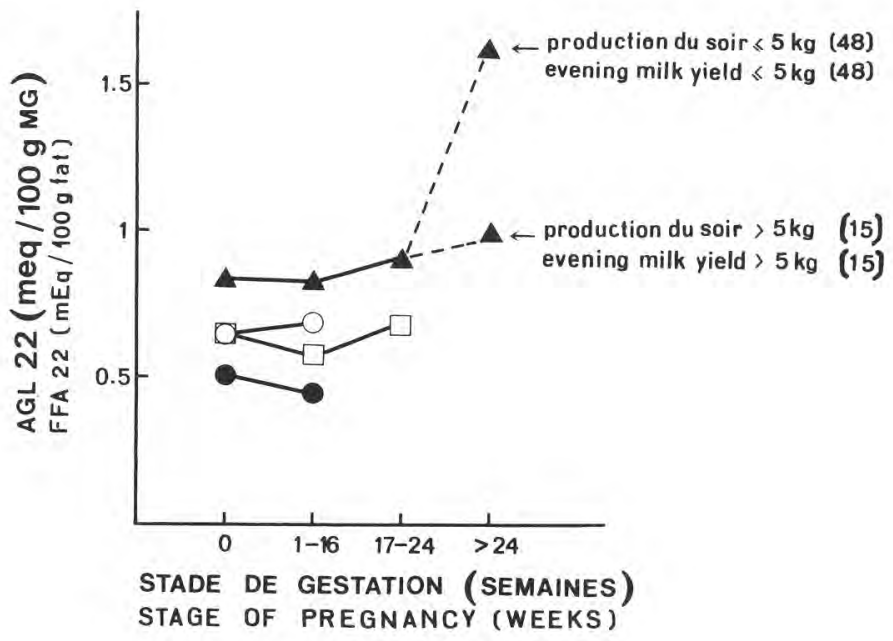

Fig. 1

Influence du stade de gestation sur la teneur en acides gras libres du lait après $22 \mathrm{~h}$ de stockage à $4{ }^{\circ} \mathrm{C}$ (AGL22), en fonction du stade de lactation. Semaines de lactation (nombre de données par point) :

- $1-12(45-9)$;

O, 13-24 (58-119) ;

$\square, 25-32(11-37-43)$;

$\mathbf{\Delta},>32$ semaines (15-17-37-63).

Influence of pregnancy stage on free fatty acid content in milk after $22 \mathrm{~h}$ storage at $4{ }^{\circ} \mathrm{C}$ (FFA-22) according to stage of lactation. Weeks of lactation (number of data for each point) :

- 1-12 (45-9) ;

O, 13-24 (58-119) ;

$\square, 25-32(11-37-43)$;

$\mathbf{\Delta},>32$ weeks $(15-17-37-63)$. 
B. Effets du stade de lactation, du stade de gestation et du niveau de production sur les teneurs en $A G L$ du lait

Les effets respectifs du stade de lactation, du stade de gestation et du niveau de production sur l'augmentation des teneurs en AGL au cours de la lactation ont pu être dissociés.

1. La hausse des teneurs en AGL22 en fin de lactation résulte d'un effet combiné du stade de gestation avancé ( $>24$ semaines) et du faible niveau de production laitière $(<5 \mathrm{~kg}$ à la traite du soir). En effet, sur les prélèvements de lait effectués après 32 semaines de lactation (tabl. 2, analyse II et fig. 1),

\section{TABLeAu 2}

Effets du stade de lactation, du stade de gestation et du niveau de production laitière à la traite du soir sur les teneurs en AGL avant ( $A G L$ initiaux) et après 22 h de stockage à $4{ }^{\circ} \mathrm{C}(A G L 22)$ du lait de vache

\begin{tabular}{|c|c|c|c|c|c|c|c|}
\hline $\begin{array}{l}\text { Analyse (1) } \\
\text { Analysis (1) }\end{array}$ & \multicolumn{7}{|c|}{ I } \\
\hline $\begin{array}{l}\text { Prélèvements } \\
\text { Samples } \\
\left(\mathrm{n}, \mathrm{p}^{2}\right)\end{array}$ & \multicolumn{7}{|c|}{$\begin{array}{c}\text { Ensemble } \\
\text { all } \\
(211,456)\end{array}$} \\
\hline \multirow[t]{2}{*}{$\begin{array}{l}\text { Facteur étudié } \\
\text { Effect studied }\end{array}$} & \multicolumn{3}{|c|}{$\begin{array}{l}\text { Troupeau (4) } \\
\text { Herd (4) }\end{array}$} & \multicolumn{4}{|c|}{$\begin{array}{l}\text { Stade de lactation (semaines) } \\
\text { Stage of lactation (weeks) }\end{array}$} \\
\hline & B & M & $\mathrm{T}$ & $\leqslant 12$ & $13-24$ & $25-32$ & $>32$ \\
\hline $\begin{array}{l}\mathrm{n} \\
\mathrm{p}\end{array}$ & $\begin{array}{r}46 \\
108\end{array}$ & $\begin{array}{l}100 \\
210\end{array}$ & $\begin{array}{r}65 \\
138\end{array}$ & $\begin{array}{l}54 \\
54\end{array}$ & $\begin{array}{l}160 \\
177\end{array}$ & $\begin{array}{l}92 \\
93\end{array}$ & $\begin{array}{r}99 \\
132\end{array}$ \\
\hline $\begin{array}{l}\text { AGL initiaux (3) } \\
\text { Initial FFA (3) }\end{array}$ & $\begin{array}{r}0,28^{\mathrm{a}} \\
(0,09)\end{array}$ & $\begin{array}{r}0,27^{\mathrm{a}} \\
(0,06)\end{array}$ & $\begin{array}{r}0,32^{\mathrm{b}} \\
(0,11)\end{array}$ & $\begin{array}{r}0,26^{\mathrm{a}} \\
(0,06)\end{array}$ & $\begin{array}{r}0,27^{\mathrm{a}} \\
(0,06)\end{array}$ & $\begin{array}{r}0,29^{\mathrm{b}} \\
(0,06)\end{array}$ & $\begin{array}{r}0,32^{\mathrm{b}} \\
(0,12)\end{array}$ \\
\hline $\begin{array}{l}\text { AGL22 (3) } \\
\text { FFA-22 (3) }\end{array}$ & $\begin{array}{r}0,65^{\mathrm{a}} \\
(0,40)\end{array}$ & $\begin{array}{r}0,78^{\mathrm{b}} \\
(0,66)\end{array}$ & $\begin{array}{r}0,89^{\mathrm{b}} \\
(0,70)\end{array}$ & $\begin{array}{r}0,49^{\mathrm{a}} \\
(0,16)\end{array}$ & $\begin{array}{r}0,68^{\mathrm{b}} \\
(0,30)\end{array}$ & $\begin{array}{r}0,63^{b} \\
(0,25)\end{array}$ & $\begin{array}{r}1,15^{\mathrm{c}} \\
(1,00) \\
\end{array}$ \\
\hline $\begin{array}{l}\% \text { de la variance totale expliqué } \\
\% \text { of total variance accounted for }\end{array}$ & \multicolumn{3}{|c|}{$6 \%$} & \multicolumn{4}{|c|}{$12 \%$} \\
\hline
\end{tabular}


les teneurs en AGL22 restent stables durant les 24 premières semaines de gestation. Par contre, après 24 semaines de gestation, elles augmentent fortement dans le lait des vaches produisant moins de $5 \mathrm{~kg}$ à la traite du soir $(+81 \%$ par rapport à $17-24$ semaines, $\mathrm{P}<0,05)$, mais pas dans le lait des animaux produisant plus de $5 \mathrm{~kg}$. Les variations des teneurs en AGL initiaux avec le stade de gestation ne sont pas significatives.

2. L'effet du niveau de production existe quel que soit le stade de gestation : ainsi sur les prélèvements effectués avant 24 semaines de gestation (tabl. 2, analyse III) ou à stades de gestation comparables (fig. 2), une baisse du niveau de production s'accompagne toujours d'un accroissement des teneurs en AGL initiaux et en AGL22.

Effects of lactation stage, pregnancy stage and evening milk yield on the bovine milk FFA content before (Initial FFA) and after storage at $4{ }^{\circ} \mathrm{C}$ for $22 h$ (FFA-22)

\begin{tabular}{|c|c|c|c|c|c|c|c|c|c|c|c|}
\hline \multicolumn{5}{|c|}{ II } & \multicolumn{3}{|c|}{ III } & \multicolumn{4}{|c|}{ IV } \\
\hline \multicolumn{5}{|c|}{$\begin{array}{c}>32 \text { semaines de lactation } \\
>32 \text { weeks of lactation } \\
(99,132)\end{array}$} & \multicolumn{3}{|c|}{$\begin{array}{c}\leqslant 24 \text { semaines } \\
\text { de gestation } \\
\leqslant 24 \text { weeks } \\
\text { of pregnancy } \\
(207,391)\end{array}$} & \multicolumn{4}{|c|}{$\begin{array}{l}\text { Production entre } 5 \text { et } 10 \mathrm{~kg} \\
\text { Milk yield between } 5 \text { and } 10 \mathrm{~kg} \\
(169,288)\end{array}$} \\
\hline \multicolumn{5}{|c|}{$\begin{array}{c}\text { Stade de gestation }(\text { semaines }) \times \text { niveau } \\
\text { de production }(\mathrm{kg}) \\
\text { Stage of pregnancy }(\text { weeks }) \times \text { milk } \\
\text { yield }(\mathrm{kg})\end{array}$} & \multicolumn{3}{|c|}{$\begin{array}{l}\text { Niveau de } \\
\text { production }(\mathrm{kg}) \\
\text { Milk yield }(\mathrm{kg})\end{array}$} & \multicolumn{4}{|c|}{$\begin{array}{l}\text { Stade de lactation (semaines) } \\
\text { Stage of lactation (weeks) }\end{array}$} \\
\hline \multirow{2}{*}{0} & \multirow{2}{*}{$\leqslant 16$} & \multirow{2}{*}{$17-24$} & \multicolumn{2}{|c|}{$>24$} & \multirow{2}{*}{$\leqslant 5$} & \multirow{2}{*}{$5-10$} & \multirow{2}{*}{$>10$} & \multirow{2}{*}{$\leqslant 12$} & \multirow{2}{*}{$13-24$} & \multirow{2}{*}{$25-32$} & \multirow{2}{*}{$>32$} \\
\hline & & & $>5 \mathrm{~kg}$ & $\leqslant 5 \mathrm{~kg}$ & & & & & & & \\
\hline 11 & 15 & 37 & 15 & 48 & 29 & 165 & 62 & 9 & 121 & 77 & 56 \\
\hline 15 & 17 & 37 & 15 & 48 & 31 & 271 & 89 & 15 & 132 & 78 & 63 \\
\hline $\begin{array}{c}0,34 \\
(0,08)\end{array}$ & $\begin{array}{c}0,30 \\
(0,09)\end{array}$ & $\begin{array}{c}0,33 \\
(0,18)\end{array}$ & $\begin{array}{c}0,29 \\
(0,07)\end{array}$ & $\begin{array}{c}0,33 \\
(0,11)\end{array}$ & $\begin{array}{r}0,36^{\mathrm{a}} \\
(0,17)\end{array}$ & $\begin{array}{r}0,28^{\mathrm{b}} \\
(0,07)\end{array}$ & $\begin{array}{r}0,27^{\mathrm{b}} \\
(0,06)\end{array}$ & $\begin{array}{c}0,26 \\
(0,08)\end{array}$ & $\begin{array}{c}0,27 \\
(0,06)\end{array}$ & $\begin{array}{c}0,28 \\
(0,06)\end{array}$ & $\begin{array}{l}0,29 \\
(0,09)\end{array}$ \\
\hline $\begin{array}{r}0,83^{\mathrm{a}} \\
(0,24)\end{array}$ & $\begin{array}{r}0,81^{\mathrm{a}} \\
(0,23)\end{array}$ & $\begin{array}{r}0,89^{a} \\
(0,67)\end{array}$ & $\begin{array}{r}0,98^{\mathrm{a}} \\
(1,45)\end{array}$ & $\begin{array}{r}1,61^{b} \\
(1,20)\end{array}$ & $\begin{array}{r}0,95^{\mathrm{a}} \\
(0,57)\end{array}$ & $\begin{array}{r}0,70^{\mathrm{b}} \\
(0,33)\end{array}$ & $\begin{array}{r}0,52^{c} \\
(0,15)\end{array}$ & $\begin{array}{r}0,50^{\mathrm{a}} \\
(0,23)\end{array}$ & $\begin{array}{r}0,71^{\mathrm{b}} \\
(0,31)\end{array}$ & $\begin{array}{r}0,64^{\mathrm{b}} \\
(0,24)\end{array}$ & $\begin{array}{r}0,85^{\mathrm{b}} \\
(0,80)\end{array}$ \\
\hline \multicolumn{5}{|c|}{$10 \%$} & \multicolumn{3}{|c|}{$7 \%$} & \multicolumn{4}{|c|}{$5 \%$} \\
\hline
\end{tabular}

(1) All analyses take into account the * herd s factor. The covariate is milk fat content.

(2) $(n, p):$ (no. of cows, no. of samples).

(3) Mean, s.d. in parenthesis, in meq/100 $\mathrm{g}$ fat. Values in the same horizontal row with different superscripts differ $(P<0.05)$ for the effect studied.

(4) Herd: B, Bourg-en-Bresse; M, Marcenat; T, Theix. 


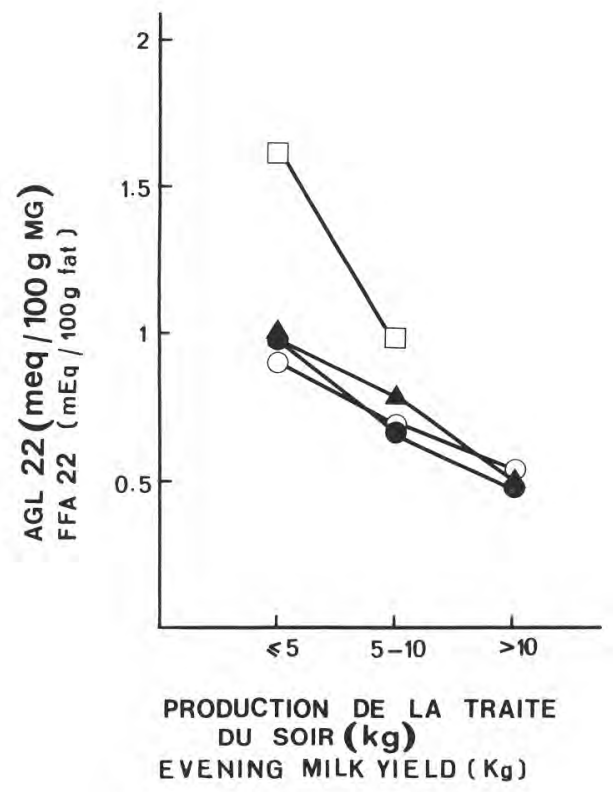

Fig. 2

Effet du niveau de production à la traite du soir sur la teneur en acides gras libres du lait après $22 \mathrm{~h}$ de stockage à $4{ }^{\circ} \mathrm{C}$ (AGL22) en fonction du stade de gestation. Semaines de gestation (nombre de données par point) :

non gravides (10-69-49);

1-16 (10-138-35) ;

$\Delta 17-24(11-64-5)$;

$>24(48-15)$.

Effect of evening milk yield on free fatty acid content in milk after $22 \mathrm{~h}$ storage at $4{ }^{\circ} \mathrm{C}$ (FFA-22) according to stage of pregnancy. Weeks of pregnancy (number of data for each point) :

non pregnant (10-69-49) ;

1-16 (10-138-35) ;

$\Delta 17-24(11-64-5)$;

$\square>24(48-15)$.

3. Le niveau de production et le stade de lactation sont fortement liés $(\mathrm{r}=0,70, \mathrm{n}=456)$. Afin de séparer les effets de ces 2 facteurs, l'analyse IV a été faite sur les prélèvements correspondant à des productions comprises entre 5 et $10 \mathrm{~kg}$. Elle a montré un effet propre du stade de lactation sur les teneurs en AGL22 du lait qui augmentent significativement après 12 semaines de lactation $(\mathrm{P}<0,05)$. Il n'y a par contre pas d'effet du stade de lactation proprement dit sur les teneurs en AGL initiaux.

En résumé, dans les 3 troupeaux, le niveau de production a un effet sur les teneurs en AGL22 quels que soient le stade de lactation et le stade de gestation des animaux. L'augmentation des teneurs en AGL22 en fin de 
lactatation est essentiellement due à un effet combiné du stade avancé de gestation ( $>24$ semaines) et du faible niveau de production $(<5 \mathrm{~kg})$. Il existe toutefois un effet propre du stade de lactation, les teneurs en AGL 22 étant plus faibles avant 12 semaines de lactation. Par contre, seule la diminution du niveau de production contribue à l'augmentation des teneurs en AGL initiaux au cours de la lactation.

Par ailleurs, les parts de variance des teneurs en AGL22 expliquées par les facteurs stade de lactation, stade de gestation et niveau de production sont faibles $(10 \%$ par les effets combinés du stade de gestation et du niveau de production, analyse II ; $7 \%$ par l'effet du niveau de production, analyse III ; $5 \%$ par l'effet du stade de lactation, analyse IV, voir tableau 2), la part des variations individuelles étant très importante (Chazal et Chilliard, 1987b).

\section{Discussion}

La teneur en AGL22 du lait représente la somme des AGL initiaux et des produits de la lipolyse spontanée au cours du stockage au froid. Les teneurs en AGL initiaux, positivement corrélées à celles en AGL22 $(r=0,35, n=456$, $\mathrm{P}<0,01$ ), subissent de faibles variations (moyenne de $0,29 \pm 0,09 \mathrm{meq} / 100 \mathrm{~g}$ MG sur 456 échantillons) contrairement aux teneurs en AGL22 (0,78 $\pm 0,63$ meq/100 g MG). Ainsi la lipolyse spontanée (AGL22 - AGL initiaux) est fortement corrélée à la teneur en AGL22 du lait $(\mathrm{r}=0,99, \mathrm{n}=456, \mathrm{P}<0,01)$, dont elle suit les variations.

\section{A. Variations des teneurs en $A G L$ du lait selon le troupeau}

Les variations des teneurs en AGL du lait en fonction du troupeau ne sont pas liées à un éventuel effet de la race des animaux. En effet, aucune différence de teneurs en AGL initiaux d'une part et en AGL22 d'autre part n'a été mise en évidence entre les races Frisonne Pie-Noire et Montbéliarde (Chazal et Chilliard, 1987a).

Les teneurs en AGL initiaux dans les laits des animaux du troupeau de Theix, supérieures à celles mesurées dans ceux des troupeaux de Bourg-enBresse et de Marcenat, peuvent être liées aux teneurs plus élevées en leucocytes dans les laits de ce troupeau (tabl. 1). Il existe en effet une corrélation positive significative entre les teneurs en AGL initiaux et en leucocytes $(\mathrm{r}=0,34, \mathrm{P}<0,01$, pour 398 laits provenant de 270 vaches de 4 troupeaux $)$ en accord avec les résultats de TARASSUK et YAgughi (1958), TALlamy et RaNDOLPH (1969), Salih et Anderson (1979). Ce taux plus élevé de cellules dans les laits de Theix peut être expliqué par l'âge plus élevé des animaux (4 lactations en moyenne, contre 2,8 à Marcenat et 2,3 à Bourg-en-Bresse) et à la fréquence plus grande des mammites subcliniques dans ce troupeau, liée à un ralentissement des réformes consécutif à la reconstitution de ce troupeau.

Les teneurs en AGL22 plus faibles à Bourg-en-Bresse qu'à Theix et Marcenat, indépendamment du stade de lactation et du niveau de production 
des animaux, pourraient être liées à l'intervalle entre traite du matin et traite du soir plus élevé à Bourg-en-Bresse $(10 \mathrm{~h} 30)$ qu'à Theix $(8 \mathrm{~h} 30)$ ou à Marcenat (9 h 30). En effet, SuHren et al. (1981) ont montré une augmentation sensible de lipolyse dans le lait recueilli le soir lorsque l'intervalle avec la traite du matin diminue, surtout en dessous de $10 \mathrm{~h}$.

B. Variations des teneurs en $A G L$ du lait au cours de la lactation : effet du stade de lactation, du stade de gestation et du niveau de production

Les augmentations des teneurs en AGL initiaux et en AGL22 au cours de la lactation sont en accord avec les résultats de SALIH et ANDERSON (1979a), Ahrne et Bjorck (1985) et Chazal et Chilliard (1986).

Comme dans l'étude de Chazal et Chilliard (1986), les teneurs en AGL22 augmentent après 12 semaines et surtout après 32 semaines de lactation, lorsque le stade de gestation est supérieur à 24 semaines et le niveau de production laitière à la traite du soir inférieur à $5 \mathrm{~kg}$.

Nous confirmons donc ici sur 3 troupeaux et 3 races l'influence nette du stade de gestation sur la lipolyse du lait. Ceci a été aussi confirmé au cours de la saison 1984-1985 sur le troupeau Montbéliard de la Côte-Saint-André (résultats non publiés) et très récemment dans une courte communication de BACHMAN et al. (1986) sur vaches Holstein et Jersey, ainsi que dans une enquête de terrain sur laits de tank effectuée en 1985-1986 par le G.I.E. laitviande Rhône-Alpes.

L'effet du stade de gestation sur l'augmentation de lipolyse en fin de lactation pourrait résulter de l'augmentation des œstrogènes circulants durant la $2^{\mathrm{C}}$ moitié de la gestation (M. Terqui, cité par Thatcher et al., 1979), compte tenu de l'effet favorable d'injections d'œstrogènes sur la lipolyse (KASTlI, 1973 ; BaChMAN, 1982 ; Heo et al., 1983), qui augmentent la quantité de lipase liée à la crème (BACHMAN, 1982) ou induisent l'apparition d'un nouveau système lipolytique (Heo et al., 1983).

Toutefois, les importances relatives du stade de gestation et du niveau de production dans cet essai sont différentes de celles qui avaient été observées dans l'étude de Chazal et Chilliard (1986). En effet, nous observons ici un effet prépondérant du niveau de production quel que soit le stade de gestation, alors que dans l'essai précédent, l'effet du stade de gestation était prépondérant quel que soit le niveau de production.

Cette différence pourrait être liée à la structure des populations étudiées dans les 2 essais. Le présent essai porte sur 211 vaches de 3 troupeaux suivies seulement 2 à 3 fois au cours de leur lactation. Les variations individuelles sont très importantes et peuvent masquer l'effet du stade de gestation. L'essai de Chazal et Chilliard (1986) portait sur 57 vaches d'un seul troupeau suivi 14 à 20 fois au cours de la lactation. Les variations individuelles sont donc réduites, pour un nombre de données 2 fois plus important (942 données contre 546 dans cet essai). L'effet du stade de gestation a pu ainsi être mis en évidence plus aisément.

A mêmes stades de lactation et de gestation, les différences dues au niveau de production résultent d'un ensemble de facteurs tels que le génotype, 
le potentiel laitier, les conditions alimentaires et sanitaires, l'intervalle de traite... qui sont d'autant plus variables que le nombre de troupeaux et d'animaux étudiés augmente. Ceci explique peut-être l'effet prépondérant du niveau de production dans la présente étude.

L'augmentation de lipolyse avec la baisse de production laitière est d'autant plus élevée que les animaux sont gravides depuis plus de 24 semaines. Ceci pourrait être dû à une plus grande sensibilité du substrat à l'induction mécanique de lipolyse en fin de gestation : en effet, à ce stade, le taux butyreux augmente $(48,0 \mathrm{~g} / 1$ après 24 semaines de gestation, contre $42,4 \mathrm{~g} / \mathrm{l}$ avant 16 semaines) et la taille des globules gras diminue (MULDER et WALSTRA, 1974) alors que, parallèlement, les phospholipides du lait (le matériel membranaire) n'augmentent pas (Kinsella et Houghton, 1975).

Une baisse du niveau de production pourrait entraîner une baisse du flux de lait pendant la traite, pouvant induire de la lipolyse (FLEMING, 1979). Toutefois, à Theix, aucune liaison n'a été observée entre vitesse de traite (kg lait/minute) et teneur en AGL22 du lait, mesurées à la traite du soir de 31 vaches à $38 \pm 6$ semaines de lactation $(\mathrm{r}=0,01, \mathrm{n}=31)$.

Les effets du stade de gestation et du niveau de production n'expliquent qu'un faible pourcentage de la variance totale de la lipolyse en fin de lactation. Toutefois, la variance des teneurs en AGL22 en fin de lactation est très élevée (42 fois plus importante après 32 semaines qu'avant 12 semaines de lactation), ce qui explique en partie le faible pourcentage de variance expliqué par des facteurs physiologiques dont l'importance est indéniable, d'autant plus qu'ils peuvent agir en synergie avec d'autres facteurs, alimentaires ou individuels (Chazal et Chilliard, 1986, 1987a, 1987b ; Chazal et al., 1987).

\section{Conclusion}

Cette étude confirme sur 3 troupeaux et 3 races les résultats obtenus sur le troupeau de Montbéliard sur La Côte-Saint-André (Isère), qui montraient pour la première fois un effet du stade de gestation sur l'augmentation de la lipolyse du lait de vache en fin de lactation (Chazal et Chilliard, 1986). Elle précise et nuance toutefois ces résultats, puisque les modalités de l'étude (rapport entre le nombre d'individus et le nombre de prélèvements par individu au cours de la lactation) semblent modifier les importances relatives des effets du stade de gestation et du niveau de production. Cependant un stade avancé de gestation semble toujours engendrer un état prédisposant à la lipolyse, qui se développerait dans certaines conditions favorisantes telles que des niveaux de production faibles. En pratique, les 2 mois précédant le tarissement des animaux sont critiques pour la lipolyse, surtout lorsque le niveau de production de lait devient inférieur à $5 \mathrm{~kg}$ à la traite du soir et dans le cas de vêlages groupés. 


\section{Remerciements}

Nous remercions J.P. Garel, A. OLLIER et J. DEMULE, responsables de la gestion des 3 troupeaux expérimentaux, Frédérique GIGNIAC, Florence TRINCAT et Ginette BovQUET qui ont effectué les dosages d'AGL et M.C. SINSARD pour la dactylographie du manuscrit. Ce travail a été réalisé grâce au soutien financier du Ministère de la Recherche et de la Technologie, et de l'Institut Technique de l'Elevage Bovin.

Reçu le 20 mai 1986. Accepté pour publication le 7 décembre 1986.

\section{Références bibliographiques}

AhrNe L., BJörCK, 1985. Lipolysis and the distribution of lipase activity in bovine milk in relation to stage of lactation and time of milking, J. Dairy Res., 52, 55-64.

BACHMAN K.C., 1982. Effect of exogenous estradiol and progesterone upon lipase activity and spontaneous lipolysis in bovine milk. J. Dairy Sci., 65, 907-914.

Bachman K.C., Hayen M.J., Morse D., Wilcox C.J., 1986. Effect of pregnancy upon acid degree values of bovine milk fat (Abstract). J. Dairy Sci., 69, (suppl. 1), 62.

Chazal M.P., Cartier P., Chilliard Y., Flechet J., Bauchart D., Duboisset F., Meyer M., 1984. Dosage des acides gras libres du lait par la méthode BDI. Cah. Tech. INRA, (6), 17-27.

Chazal M.P., Chilliard Y., 1986. Effect of stage of lactation, stage of pregnancy, milk yield and herd management on seasonal variation in spontaneous lipolysis in bovine milk. J. Dairy Res., 53, 529-538.

Chazal M.P., Chilliard Y., 1987a. Effect of breed of cow (Friesian and Montbéliarde) on spontaneous and induced lipolysis in milk. J. Dairy Res., 54, 7-11.

Chazal M.P., Chilliard Y., 1987b. Les variations individuelles de la lipolyse spontanée du lait de vache : effet du numéro de lactation et répétabilité au cours de 2 lactations successives. Lait, 67 , sous presse.

Chazal M.P., Chilliard Y., Coulon J.B., 1987. Effect of nature of forage on spontaneous lipolysis in milk from cows in late lactation. J. Dairy Res., 54, 13-18.

Chilliard Y., 1982. Variations physiologiques des activités lipasiques et de la lipolyse spontanée dans les laits de vache, de chèvre et de femme : Revue bibliographique. Lait, 62, 1-31 et 125154.

FLeming M.G., 1979. Lipolysis in bovine milk as affected by mechanical and temperature activation. A review. Ir. J. Food Sci. Technol., 3, 111-129.

Freeden H., Bowstead J.E., Dunkley W.L., Smith L.M., 1951. Hydrolytic rancidity in milk. II. Some management and environmental factors influencing lipolysis. J. Dairy Sci., 34, 521-528.

Heo T.R., Amado R., Bachmann M.R., 1983, Beitrag zur Kenntnis des Lipasesystemes der Milch. Schweiz. Milchw. Forschung, 12, 59-65.

Jellema A., 1980. Physiological factors associated with lipolytic activity in cow's milk. Bull. Fed. Int. Lail., Doc. 118, 36-40.

JENSEN R.G., 1959. Spontaneous lipolysis in milk throughout the stages of lactation of individual cows. J. Dairy Sci., 42, 1619-1621.

KästLı P., 1973. Der Einfluss von Ostrogenen und hypophysären Gonadotropinen auf die Milch. Wien. Tieraerzil. Mschr., 60, 43-46.

Kinsella J.E., Houghton G., 1975. Phospholipids and fat secretion by cows on normal and low fiber diets : lactational trends. J. Dairy Sci., 58, 1288-1293.

Mulder H., Walstra P., 1974. The milk fat globule. Technical communication no. 4 of the Commonwealth Bureau of Dairy Science and Technology, Universities Press, Belfast. 296. 
Salih A.M.A., Anderson M., 1979. Observations on the influence of high cell count on lipolysis in bovine milk. J. Dairy Res., 46, 453-462.

Salih A.M.A., Anderson M., 1979a. Effect of diet and stage of lactation on bovine milk lipolysis. J. Dairy Res., 46, 623-631.

SEebeck R.M., 1973. The effect of body weight loss on the composition of brahman cross and africander cross steers. I. Empty body weight, dressed carcass weight and offal components. $J$. Agric. Sci., 80, 201-210.

Suhren G., Hamann J., Heeschen W., Tolle A., 1981. Zum Einfluss tierindividueller Faktoren, der Gemelksfraktion und des Melkzeit-intervalls auf den Gehalt freier Fettsäuren (FFA) in Rohmilch. Milchwissenschaft, 36, 150-153.

Tallamy P.T., RandolPh H.E., 1969. Influence of mastitis on properties of milk. IV. Hydrolytic rancidity. J. Dairy Sci., 52, 1569-1572.

Tarassuk N.P., Yaguchi M., 1958. Effect of mastitis on susceptibility of milk to lipolysis. $J$. Dairy Sci., 41, 1482 (Abstract).

Thatcher W.W., Wilcox C.J., Bazer F.W., Collier R.J., Eley R.M., Stover D.G., Bartol F.F., 1979. Bovine conceptus effects prepartum and potential carryover effects postpartum. In : Beltsville Symposia in Agricultural Research. 3. Animal Reproduction. Ed. John Wiley and Sons, New York, 259-275. 